\title{
Anterior Gradient Protein 2 Promotes Mucosal Repair in Pediatric Ulcerative Colitis
}

\author{
Xiaolin Ye, ${ }^{1}$ Jie Wu $\mathbb{D}^{1},{ }^{1}$ Jing $\mathrm{Li}^{2}$ and Hongyu Wang ${ }^{2}$ \\ ${ }^{1}$ Department of Gastroenterology, Beijing Children's Hospital, Capital Medical University, National Center for Children's Health, \\ Beijing 100045, China \\ ${ }^{2}$ Department of Pediatrics, China Medical University Affiliated with Shengjing Hospital, Shenyang, 110004 Liaoning, China
}

Correspondence should be addressed to Jie Wu; wujiedoc@163.com

Received 25 August 2020; Accepted 5 May 2021; Published 18 May 2021

Academic Editor: Hon W. Koon

Copyright () 2021 Xiaolin Ye et al. This is an open access article distributed under the Creative Commons Attribution License, which permits unrestricted use, distribution, and reproduction in any medium, provided the original work is properly cited.

\begin{abstract}
Mucosal healing comprises a key goal of ulcerative colitis (UC) treatment. Anterior gradient protein 2 (AGR2) plays an important role in maintaining intestinal homeostasis in UC. However, the role of AGR2 in the repair of mucosal injury is not yet clear. This study is aimed at investigating the expression of AGR2 in the intestinal tissues of children with UC and its role in repairing mucosal injury. Forty UC patients who were hospitalized in the Pediatric Gastroenterology Ward of Shengjing Hospital affiliated with China Medical University between July 1, 2013, and May 31, 2020, and 20 children who had normal colonoscopy results during the same period (control group) made up the study sample. The disease activity of UC was evaluated based on the pediatric ulcerative colitis activity index, and the ulcerative colitis endoscopic index was evaluated according to the Rachmilewitz score. Immunohistochemical staining was employed to examine the differences in AGR2 expression in the intestinal mucosa between groups. The protective effect of AGR2 in a model of tumor necrosis factor-alpha- (TNF- $\alpha$-) induced intestinal mucosal barrier injury and the underlying molecular mechanism were explored through in vitro experiments. The results showed that compared with the normal control group, UC patients in the remission or active period had significantly higher expression of AGR2 in the intestine. AGR2 expression was positively correlated with Ki67, an intestinal epithelial cell proliferation marker, but negatively correlated with the degree of endoscopic mucosal injury. In an in vitro model, AGR2 overexpression promoted cell proliferation and migration and inhibited TNF- $\alpha$-induced intestinal epithelial barrier damage by activating yes-associated protein (YAP). Collectively, our study suggests that AGR2 might serve as a valuable biomarker to help assess the condition and mucosal healing status of UC patients. In vitro, AGR2 promoted the repair of intestinal mucosal barrier injury by activating YAP.
\end{abstract}

\section{Introduction}

The intestinal epithelial barrier is continuously exposed to an environment consisting of commensal microorganisms, food antigens, and invading pathogens [1]. Defective functioning of the intestinal epithelial barrier will cause microbial translocation and infiltration of inflammatory factors, eventually leading to inflammatory bowel disease (IBD) $[2,3]$. Mucosal healing is a key factor in promoting disease remission and prolonging nonoperative survival in IBD patients [4]. To restore the epithelial barrier and gastrointestinal function, a rapid and effective way of repairing mucosal damage is needed. The rapid proliferation of intestinal epithelial cells and migration of intestinal epithe- lial cells to the injured sites are considered the key factors that promote mucosal healing $[5,6]$.

Anterior gradient protein 2 (AGR2), a member of the protein disulfide isomerase (PDI) family, plays an important role in maintaining intestinal homeostasis in IBD. AGR2knockout mice show spontaneous diarrhea and goblet cell dysfunction, which are very similar to the symptoms and pathology of ulcerative colitis (UC) in humans [7]. A previous study conducted by our group has confirmed that AGR2 regulates the expression of tight junction proteins and inhibits inflammatory factor-mediated intestinal mucosal barrier injury [8]. However, the role of AGR2 in the repair of mucosal injury is not yet clear. By analyzing the relationship between AGR2 expression in the intestinal mucosa 
TABle 1: Patient characteristics.

\begin{tabular}{|c|c|c|c|}
\hline Variable & $\begin{array}{c}\text { Active UC } \\
(n=30)\end{array}$ & $\begin{array}{c}\text { Inactive UC } \\
\quad(n=10)\end{array}$ & $\begin{array}{l}\text { Controls } \\
(n=20)\end{array}$ \\
\hline Age (years), median (range) & $8(7-10)$ & $10(9-12)$ & $8(6-10)$ \\
\hline Sex, male, $N(\%)$ & $14(46.7)$ & $4(40.0)$ & $8(40.0)$ \\
\hline \multicolumn{4}{|l|}{ Laboratory examination } \\
\hline $\mathrm{WBC}\left(10^{9} / 1\right)$, mean $\pm \mathrm{SD}$ & $10.7 \pm 3.6$ & $6.7 \pm 1.7$ & $7.1 \pm 1.9$ \\
\hline $\mathrm{Hb}(\mathrm{g} / \mathrm{l})$, median (range) & $108(94-119)$ & $127(123-129)$ & $121(118-128)$ \\
\hline $\operatorname{PLT}\left(10^{9} / 1\right)$, mean $\pm \mathrm{SD}$ & $390 \pm 130$ & $324 \pm 56$ & $273 \pm 60$ \\
\hline Alb $(g / l)$, mean $\pm S D$ & $31.8 \pm 3.9$ & $36.9 \pm 2.7$ & $38.8 \pm 2.6$ \\
\hline CRP (mg/l), median (range) & $15.0(5.3-49.0)$ & $3.2(1.2-4.6)$ & $3.4(2.1-4.8)$ \\
\hline ESR $(\mathrm{mm} / \mathrm{h})$, median (range) & $45(18,62)$ & $12(9-16)$ & $9(8,11)$ \\
\hline \multicolumn{4}{|l|}{ Disease location, $N$} \\
\hline Proctitis/left-sided/extensive/pancolitis & $4 / 16 / 7 / 3$ & $2 / 3 / 4 / 1$ & - \\
\hline
\end{tabular}

and endoscopic disease activity in children with UC and by examining in vitro the effect of AGR2 on the proliferation, apoptosis, and migration of intestinal epithelial cells, this study clarified the role of AGR2 in mucosal healing in UC.

\section{Materials and Methods}

2.1. Research Subjects. Forty UC patients who were hospitalized in the Pediatric Gastroenterology Ward of Shengjing Hospital affiliated to China Medical University between July 1, 2013, and May 31, 2020 (including 10 UC patients in remission period and $30 \mathrm{UC}$ patients in active period), and 20 children who had normal colonoscopy results during the same period (control group) were selected as the research sample. Paraffin-embedded intestinal mucosal samples were collected from the two groups, and clinical information of the disease and disease activity scores were obtained by reviewing the clinical data of the patients. This study was approved by the ethics committee of Shengjing Hospital affiliated with China Medical University (approval no. 2020PS298K). The basic information of the research subjects is shown in Table 1.

2.1.1. Inclusion Criteria for UC Patients. The inclusion criteria were age younger than 14 years and a diagnosis of UC under the criteria of the "Expert Consensus on the Diagnosis and Treatment of Pediatric Inflammatory Bowel Diseases" jointly formulated by the digestive group and the clinical nutrition group of the Pediatric Branch of the Chinese Academy of Medical Sciences [9]. The exclusion criteria were concurrent infectious diseases or other autoimmune diseases.

2.1.2. Inclusion Criteria for the Control Group. The inclusion criteria were age younger than 14 years, being physically fit, and normal colonoscopy results, routine blood test results, C-reactive protein (CRP) level, erythrocyte sedimentation rate (ESR), and liver/kidney function. The exclusion criteria were concurrent abnormalities in the nervous system, respiratory system, endocrine system, or hematopoietic system and long-term use of nonsteroidal anti-inflammatory drugs, hormones, or proton pump inhibitors.

2.2. Endoscopic Manifestations and Disease Activity Assessment in Patients with UC. According to the Paris classification [10], patients with UC were classified into the E1 type (ulcerative proctitis), E2 type (left hemicolitis), E3 type (diffuse colitis), and E4 type (total colitis) based on the degree of disease involvement. The pediatric ulcerative colitis activity index (PUCAI) was used to assess disease activity. A PUCAI score $<10$ was defined as remission, $10-34$ as mild activity, 35-64 as medium activity, and $\geq 65$ as heavy activity. The Rachmilewitz endoscopic scoring system [11] was used to quantify the endoscopic colonic disease activity in UC patients. The specific scoring criteria were as follows: [1] granular sensation: absent, 0 points; present, 2 points; [2] blood vessel distribution: normal, 0 points; blurry and disordered, 1 point; completely absent, 2 points; [3] mucosal fragility: none, 0 points; slightly increased, 2 points; significantly increased, 4 points; [4] mucosal damage: absent, 0 points; mild, 2 points; significant, 4 points. A score of $0-3$ points defined remission and mucosal healing, whereas a score of 4-12 points defined active disease.

2.3. Immunohistochemical Staining. Immunohistochemical staining was performed as described [12]. Paraffin sections were first dewaxed and hydrated, then subjected to sodium citrate/microwave oven-based antigen retrieval. The sections were naturally cooled at room temperature and then soaked three times in phosphate-buffered saline (PBS) for $5 \mathrm{~min}$ each. Then, the sections were incubated with $3 \%$ $\mathrm{H}_{2} \mathrm{O}_{2}$ at room temperature for $15 \mathrm{~min}$ to eliminate endogenous peroxidase activity. After being soaked three times in PBS ( 5 min each), the sections were incubated with normal goat serum at room temperature for $15 \mathrm{~min}$. Primary antibodies (anti-AGR2 antibody, Abcam, 1:200 dilution; and anti-Ki67 antibody, Wanlei, 1:200 dilution) were added dropwise until the tissues were completely covered. The tissue sections were then placed in a wet box and incubated at $4^{\circ} \mathrm{C}$ overnight. After three soaks in PBS 
( 5 min each), the tissue sections were incubated with secondary antibodies at room temperature for $60 \mathrm{~min}$. The sections were again soaked three times in PBS for $5 \mathrm{~min}$ each, colorized with $3,3^{\prime}$-diaminobenzidine (DAB), counterstained with hematoxylin, and rinsed with running water for $20 \mathrm{~min}$. The blue-dyed sections were dehydrated, cleared, and mounted. The sections were then imaged under 400x magnification.

2.4. Analysis of the Immunohistochemical Results. All pathological sections were scored independently by two experienced pathologists. Semiquantitative analysis was performed based on a combination of immunohistochemical score and percentage of specifically stained cells, as described previously [13]. The staining intensity was graded as follows: 0 points, not yellow; 1 point, light yellow; 2 points, brownishyellow; 3 points, brown. The percentage of positively stained cells was scored as follows: 0 points, $<5 \%$ positive cells; 1 point, $6-25 \%$; 2 points, $26-50 \%$; 3 points: $51-75 \%$; 4 points, $>75 \%$. The total score was calculated by multiplying the score of staining intensity by the score of the percentage of positively stained cells. A total score of greater than 4 points was defined as immunohistochemically positive staining.

2.5. Cell Culture and Monolayer Preparation. Caco-2 cells, obtained from the Cell Bank of the Chinese Academy of Sciences, were grown in RPMI-1640 medium supplemented with $10 \%$ fetal bovine serum (ScienCell Research Laboratories, San Diego, CA, USA) at $37^{\circ} \mathrm{C}$ in an incubator with $5 \%$ $\mathrm{CO}_{2}$. After the cell confluence reached $80 \%$ or greater, the cell density was adjusted, a cell count was performed, and the cells were further diluted to $3 \times 10^{4} / \mathrm{ml}$ and inoculated into Transwell filters with a pore size of $0.4 \mu \mathrm{m}$ (Millipore, Billerica, MA, USA). The medium was changed every other day until the monolayer was prepared, as described in a previous study [14].

2.6. Plasmid Transfection. Caco-2 monolayers were transfected with a pcDNA3.1-AGR2 plasmid and a pcDNA3.1 vector control plasmid using Lipofectamine 2000 (Invitrogen, Carlsbad, CA, USA) according to the manufacturer's instructions. Opti-MEM Medium (100 $\mu$ l) (Invitrogen) and $8 \mu \mathrm{l}$ of Lipofectamine 2000 (Invitrogen) were incubated at room temperature for $5 \mathrm{~min}$. Additionally, $100 \mu \mathrm{l}$ of OptiMEM Medium and $2 \mu \mathrm{g}$ of plasmid were incubated at room temperature for $5 \mathrm{~min}$. The solutions were mixed and placed at room temperature for $20 \mathrm{~min}$ until the resulting solution was well mixed. The mixture was added to a six-well plate and cultured for $4 \mathrm{~h}$. Then, $48 \mathrm{~h}$ after transfection, the cells were harvested for further study, including Western blotting and $\mathrm{qPCR}$ analyses.

2.7. Determination of Transepithelial Electrical Resistance (TEER). Caco-2 cells were seeded in Transwell chambers with a $0.4 \mu \mathrm{m}$ pore size (Millipore, Billerica, MA, USA) at a density of $3 \times 10^{4}$ cells $/ \mathrm{ml}$, and the TEER was assayed using an epithelial voltohmmeter (Millicell-ERS ${ }^{\circledR}$, Millipore, Billerica, MA, USA) according to the method described in a previous study [15]. Two Transwell wells with culture medium only were used as blank control wells. The entire measurement process was carried out at a constant temperature. Three points in different directions in each Transwell were continuously measured three times each. The average value was recorded as the measured TEER.

$\operatorname{TEER}\left(\Omega \quad \cdot \mathrm{cm}^{2}\right)=($ measured TEER - control TEER $) \times$ effective membrane area of the cell culture well.

2.8. Measurement of Flux of the Paracellular Marker Fluorescein Isothiocyanate- (FITC-) Dextran 40 (FD-40) $(40 \mathrm{kDa})$. Paracellular permeability was determined using FITC-dextran $(80 \mu \mathrm{g} / \mathrm{ml})$ (Sigma-Aldrich, St. Louis, MO, USA) according to the method described in a previous study $[16,17]$. The fluorescence value (excitation wavelength: $427 \mathrm{~nm}$, emission wavelength: $536 \mathrm{~nm}$ ) was measured with a microplate analyzer. The yellow fluorescence yellow level was calculated according to the standard curve.

Fluorescence transmittance $(\%)=$ FITC concentration in the lower chamber/FITC-dextran concentration added to the upper chamber $\times 100$.

2.9. Western Blot Analysis. Total protein was extracted by RIPA and phenylmethane sulfonyl fluoride (PMSF) lysate buffers. The protein concentrations were determined using a BCA protein assay kit (Thermo Scientific, Rockford, IL, USA). The procedure was performed as previously described [18]. The blots were incubated with primary antibodies, including anti-YAP (Abcam, 1:1000 dilution), anti-p-YAP Ser127 (Abcam, 1:2000 dilution), anti-cyclin D (Santa Cruz Biotechnology, $1: 1000$ dilution), or anti- $\beta$-actin $(1: 2000$, Proteintech, Wuhan, China), at $4^{\circ} \mathrm{C}$ overnight. The membranes were then incubated with secondary antibodies for $2 \mathrm{~h}$. The blots were visualized using an enhanced chemiluminescence substrate kit (Thermo Fisher Scientific, Rockford, USA), and the results were analyzed with Image J software.

2.10. Immunofluorescence. Caco-2 cells were fixed with $4 \%$ paraformaldehyde for $15 \mathrm{~min}$ at room temperature. For permeabilization, the cells were incubated with $0.5 \%$ Triton X100 for $30 \mathrm{~min}$ and then blocked with 5\% goat serum after incubation with anti-YAP (Abcam, 1:200) overnight at $4^{\circ} \mathrm{C}$. Then, the cells were incubated with the secondary antibody for $1 \mathrm{~h}$ at room temperature. Images were captured by a laser scanning fluorescence microscope (TCS SP5, Leica, Germany) at 400x magnification.

2.11. Examination of Cell Proliferation Using the Cell Counting Kit 8 (CCK-8) Assay. The treated Caco-2 cells were collected, prepared into a cell suspension, and counted. The cells were seeded into 96-well plates at a density of $2 \times 10^{3}$ cells per well. Blank controls were also prepared. The final volume of each well was $100 \mu \mathrm{l}$. The cells were cultured in a $37^{\circ} \mathrm{C}, 5 \% \mathrm{CO}_{2}$ incubator. At $48 \mathrm{~h}$ after treatment with the drugs, each well of cells was overlaid with $10 \mu \mathrm{l}$ of CCK-8 and incubated for $1 \mathrm{~h}$ in the $37^{\circ} \mathrm{C}, 5 \% \mathrm{CO}_{2}$ incubator. Optical density values were measured at $450 \mathrm{~nm}$ on a microplate reader and subjected to data analysis.

2.12. Flow Cytometric Analysis of the Cell Cycle. Caco-2 cells of different experimental groups were collected after the 
establishment of the model. After centrifugation, the supernatant was collected. The cells were mixed with precooled $70 \%$ ethanol and fixed at $4^{\circ} \mathrm{C}$ for $2 \mathrm{~h}$. The fixed cells were centrifuged at $2000 \mathrm{rpm}$ for $5 \mathrm{~min}$. The resulting supernatant was discarded. The cells were collected, washed twice with PBS, and centrifuged at $2000 \mathrm{rpm}$ for $5 \mathrm{~min}$. The supernatant was again discarded. The cells were slowly and fully resuspended in staining buffer $(500 \mu \mathrm{l}$ each tube). The cells were then mixed thoroughly, first with $50 \mu \mathrm{g} / \mathrm{ml}$ propidium iodide and then with $0.1 \mathrm{mg} / \mathrm{ml}$ RNase A. After incubation at $37^{\circ} \mathrm{C}$ for $30 \mathrm{~min}$ in the dark, the cells were placed in an ice bath in the dark. The cells were subjected to flow cytometry.

2.13. Flow Cytometric Analysis of Apoptosis. The groups of cells that had been transfected and treated with drugs were cultured in six-well plates. Once reaching approximately $90 \%$ confluence, the cells were collected and centrifuged at $1500 \mathrm{rpm}$ for $5 \mathrm{~min}$. The resulting supernatant was discarded. The cells were washed twice with PBS, centrifuged at $1500 \mathrm{rpm}$ for $5 \mathrm{~min}$, and collected. The supernatant was again discarded, leaving approximately $50 \mu \mathrm{l}$ of PBS. The cells were resuspended in $500 \mu \mathrm{l}$ of binding buffer. Next, the cells were mixed first with $5 \mu \mathrm{l}$ of Annexin V-fluorescein isothiocyanate and then with $10 \mu \mathrm{l}$ of propidium iodide. After incubation for $15 \mathrm{~min}$ at room temperature in the dark, the cells were subjected to flow cytometry.

2.14. Wound Healing Assay. The cells were cultured in sixwell plates until they reached $80 \%$ confluence. Then, $1 \mu \mathrm{g} / \mathrm{ml}$ mitomycin $\mathrm{C}$ was added for $1 \mathrm{~h}$ to inhibit cell proliferation. A line was carefully scratched vertically in the middle of the plates using a $200 \mu \mathrm{l}$ pipette tip. After three washes with PBS and culturing in a serum-free medium, the wound widths were measured at 0 and $24 \mathrm{~h}$ under a microscope (Nikon Eclipse TS100, Tokyo, Japan) at 100x magnification.

2.15. Statistical Methods. Data were analyzed with SPSS 21.0 statistical software. The relationship between AGR2 expression and clinicopathology was analyzed by the chi-squared test, Fisher's exact probability method, or the rank-sum test (Mann-Whitney $U$ test). The correlation between the data was analyzed through Spearman correlation. Continuous variables from in vitro assays are expressed as mean \pm SD. Two groups were compared by the independent-sample $t$ test, while three or more groups were compared by oneway analysis of variance. The data were always derived from at least three independent experiments. A $p$ value of less than 0.05 indicated that the difference was statistically significant.

\section{Results}

3.1. AGR2 Expression Is Elevated in the Intestinal Tissues in Children with UC. To determine the clinical significance of AGR2 in the progression of UC, this study examined the expression of AGR2 in the intestinal tissues of the normal control group and the UC patients through immunohistochemical staining. In the normal control group, AGR2 was mainly expressed at a low level in the cytoplasm, and the positive expression rate was $45 \%$ (9/20). In the active-stage UC patients, AGR2 expression was significantly increased in the
TABLE 2: Relationship between AGR2 expression and clinicopathological characteristics of UC patients.

\begin{tabular}{|c|c|c|c|}
\hline \multirow{2}{*}{ Characteristics No. } & \multicolumn{2}{|c|}{ AGR2 expression level } & \multirow{2}{*}{$p$ value } \\
\hline & Negative $n=$ & Positive $n=$ & \\
\hline \multicolumn{4}{|l|}{ Age (years) } \\
\hline$<9$ years & 6 & 13 & \multirow{2}{*}{0.178} \\
\hline$\geq 9$ years & 2 & 19 & \\
\hline \multicolumn{4}{|l|}{ Sex } \\
\hline Male & 5 & 13 & \multirow{2}{*}{0.475} \\
\hline Female & 3 & 19 & \\
\hline \multicolumn{4}{|l|}{ Location } \\
\hline Proctitis & 1 & 5 & \multirow{4}{*}{0.878} \\
\hline Left-side colitis & 3 & 16 & \\
\hline Extensive & 3 & 8 & \\
\hline Pancolitis & 1 & 3 & \\
\hline \multicolumn{4}{|l|}{ Stage } \\
\hline Remission & 1 & 9 & \multirow{2}{*}{0.648} \\
\hline Active & 7 & 23 & \\
\hline \multicolumn{4}{|l|}{ DAI } \\
\hline Mild & 2 & 12 & \multirow{3}{*}{0.187} \\
\hline Moderate & 3 & 6 & \\
\hline Severe & 2 & 5 & \\
\hline
\end{tabular}

intestinal tissues. Positive staining was mainly observed at the base of intestinal mucosal crypts, and AGR2 was expressed simultaneously in the cytoplasm and the nucleus. The positive expression rate was 76.7\% (23/30) $(p<0.05$ vs. control). In the remission-stage UC patients, the positive expression rate of AGR2 in the intestinal tissues was $90 \%$ (9/10). Compared with the rate in the active-stage UC patients, the difference was not statistically significant $(p>0.05)$ (Table 2). Immunohistochemical staining for Ki67 showed that the positive expression rate of Ki67 in the intestinal tissues was $55 \%$ in the normal control group $(11 / 20)$. The expression of Ki67 in the intestinal tissues was significantly increased in the active-stage UC patients, and the positive expression rate was $83.3 \%(25 / 30)(p<0.05$ vs. control). The Ki67-positive expression rate was $90 \%(9 / 10)$ in remission-stage UC patients. There was no statistically significant difference in the positive expression rate between the active-stage UC patients and the remission-stage UC patients $(p>0.05)$ (Figure 1(b)).

Further analysis was performed to determine the relationship between AGR2 expression in the intestinal tissues and the age, sex, and lesion sites of UC patients. The results showed that there were no statistically significant differences in the expression of AGR2 between patients with different ages, sexes, and lesion sites $(p>0.05)$. The disease activity in active-stage UC patients was evaluated based on PUCAI. The results showed that there was no statistically significant difference in AGR2 expression between UC patients of different clinical grades. However, AGR2 expression was closely correlated with the endoscopic mucosal healing status. Mucosal healing in UC patients was quantified based on the Rachmilewitz endoscopic score. The results showed that 

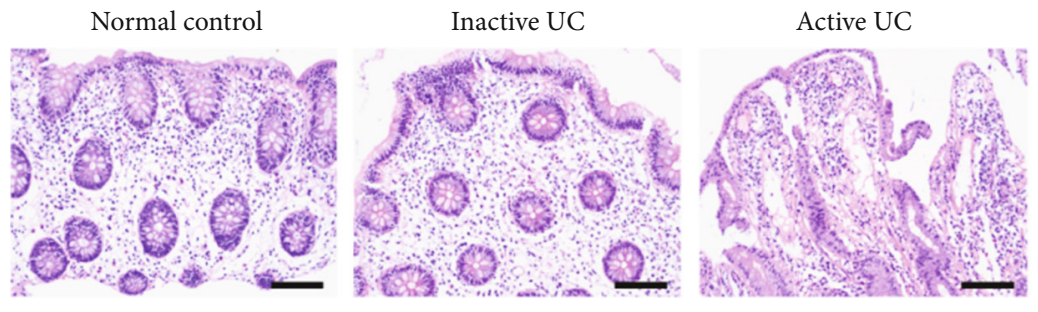

(a)
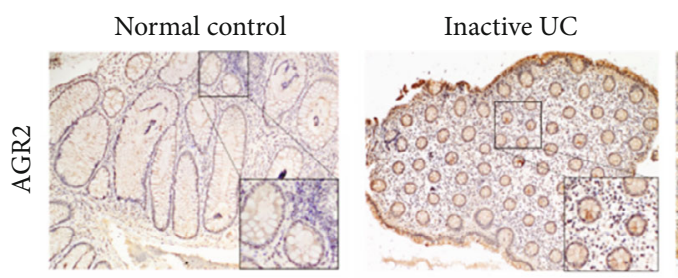

Active UC
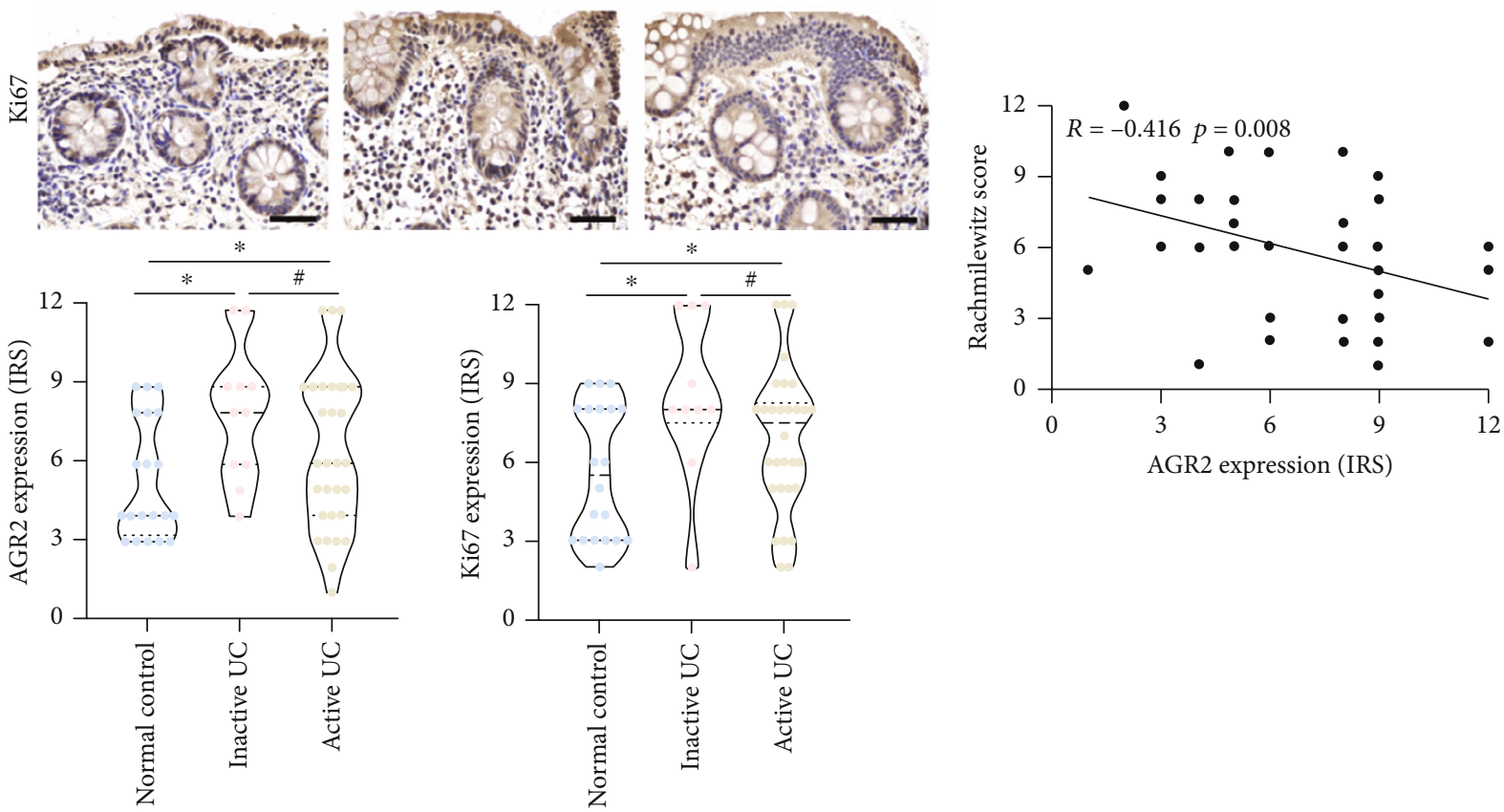

(b)

(c)

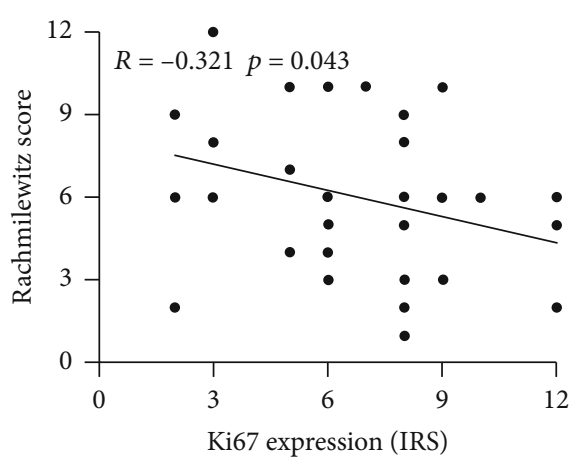

(d)

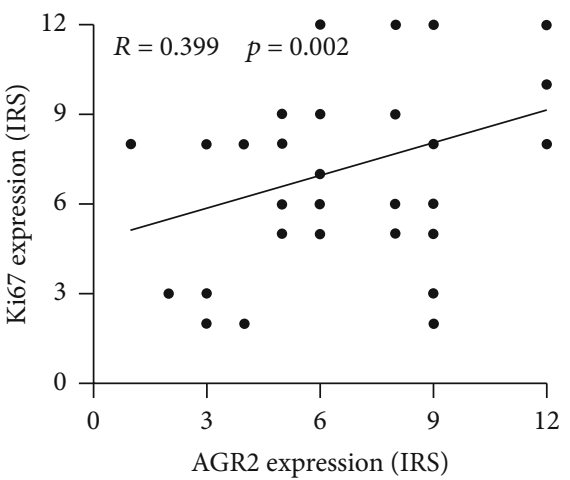

(e)

FIGURE 1: AGR2 expression is elevated in the intestinal tissues in children with UC. (a) Examination of the morphology of the intestinal tissues by HE staining. (b) Examination of the expression levels of AGR2 and Ki67 by immunohistochemical staining. (c) Spearman correlation analysis of the relationship between AGR2 expression and Rachmilewitz endoscopic score. (d) Spearman correlation analysis of the relationship between Ki67 expression and Rachmilewitz endoscopic score. (e) Spearman correlation analysis of the relationship between the expression of AGR2 and Ki67 in the intestinal mucosa epithelium of UC patients. The data are presented as the mean \pm SD, ${ }^{*} p>0.05,{ }^{*} p<0.05$. 


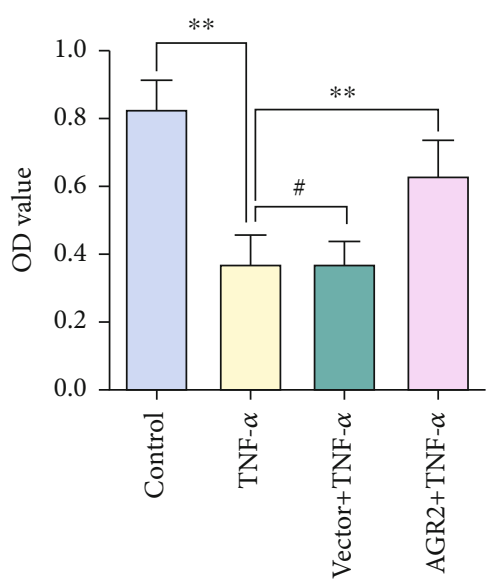

(a)
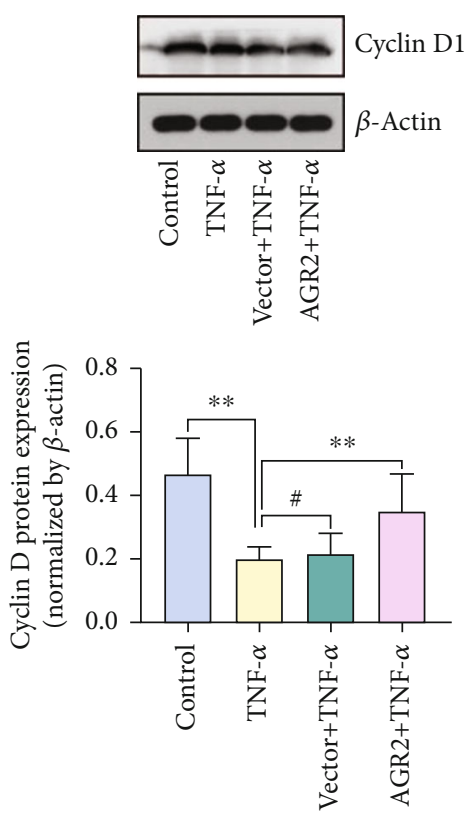

(c)
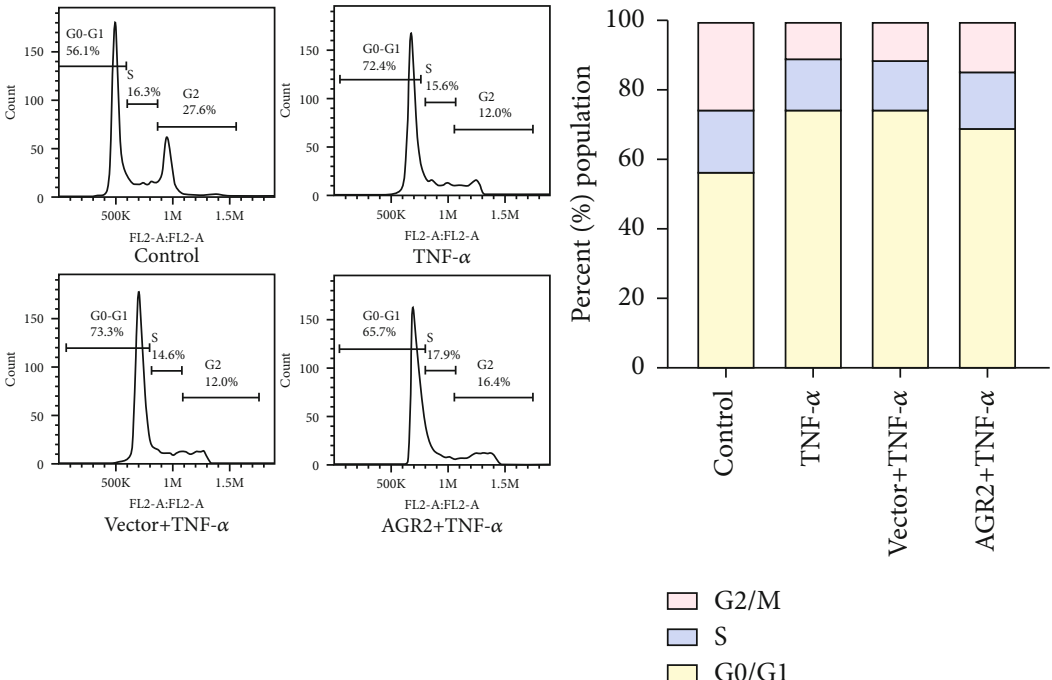

(b)
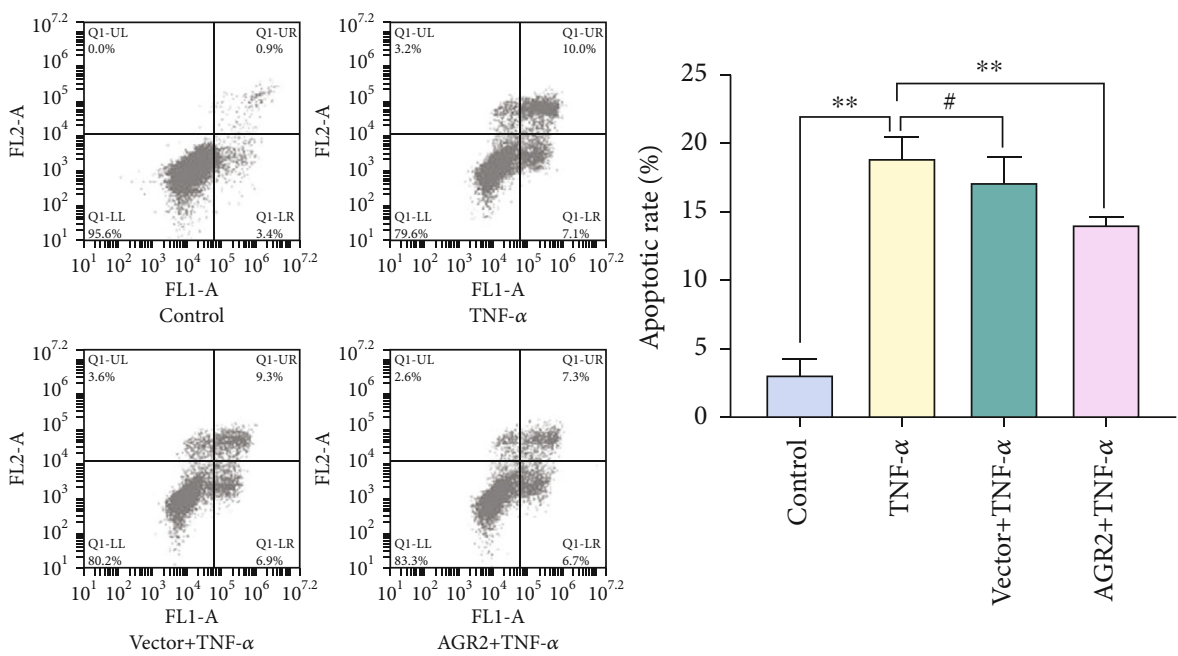

(d)

FIGURE 2: AGR2 overexpression promotes intestinal epithelial cell proliferation. (a) Viability of the Caco-2 cells as detected by CCK-8 assay. (b) The cell cycle distribution of the Caco-2 cells as determined by flow cytometry. (c) Cyclin D protein expression as determined by Western blot analysis. (d) The apoptosis level of the Caco-2 cells as determined by flow cytometry. The data are presented as the mean \pm SD, ${ }^{\#} p>0.05$, ${ }^{*} p<0.05,{ }^{* *} p<0.01$.

AGR2 expression was positively correlated with Ki67 expression in the intestinal tissues of UC patients (Figure 1(c)). In contrast, AGR2 and Ki67 expressions were negatively correlated with the Rachmilewitz endoscopic score. The more severe the intestinal mucosal injury, the lower the expression levels of AGR2 and Ki67 (Figures 1(d) and 1(e)). These results indicate that AGR2 might play a protective role on the mucosa by promoting the proliferation of intestinal epithelial cells.

\subsection{AGR2 Overexpression Promotes Intestinal Epithelial Cell} Proliferation. The AGR2 plasmid or the control plasmid was transfected into Caco- 2 cells after establishing the intes- tinal epithelial barrier model. Then, $24 \mathrm{~h}$ later, $100 \mathrm{ng} / \mathrm{ml}$ recombinant human TNF- $\alpha$ (rhTNF- $\alpha$ ) was applied to stimulate the cells in the experimental groups. Next, $48 \mathrm{~h}$ later, the CCK-8 assay was performed to examine cell proliferation. The results showed that overexpression of AGR2 significantly ameliorated the decreased growth rate induced by TNF- $\alpha$ (Figure 2(a)). To investigate the mechanism of the effect of AGR2 on intestinal epithelial cell proliferation, we used flow cytometry to determine the cell cycle distribution and apoptosis level. The results of cell cycle analysis showed that compared with the normal control group, rhTNF- $\alpha$ stimulation significantly decreased cell proliferation activity, increased the proportion of cells in G0/G1 phase, and 
decreased the proportion of cells in S and G2/M phases. Overexpression of AGR2 significantly inhibited the rhTNF$\alpha$-mediated decline in cell proliferation activity while promoting the cell cycle transition from G0/G1 to S and G2/M phases (Figure 2(b)). We further examined the expression of cell cycle-related regulatory protein cyclin D1. The results showed that the expression of cyclin D1 protein was significantly reduced in the $\operatorname{rhTNF}-\alpha$ stimulation group compared with the normal control group $(p<0.05$ ) . Pretransfection with AGR2 plasmid significantly inhibited the rhTNF- $\alpha$-induced downregulation of the cell cycle regulatory protein cyclin D1 $(p<0.05)$ (Figure $2(c))$. The above results demonstrate that AGR2 inhibits rhTNF- $\alpha$ induced G0/G1 cell cycle arrest by upregulating the expression of the cell cycle regulator cyclin D1. The results of the apoptosis analysis showed that apoptosis was significantly increased after rhTNF- $\alpha$ stimulation. In contrast, overexpression of AGR2 inhibited the rhTNF- $\alpha$-induced apoptosis (Figure 2(d)).

\subsection{AGR2 Promotes the Repair of Intestinal Mucosal Barrier} Injury by Activating YAP. YAP, a downstream effector of the Hippo pathway, plays a key role in the repair of intestinal tissue damage in UC and participates in the regulation of cell proliferation and apoptosis [19]. Under normal conditions, YAP exists in the cytoplasm in the phosphorylated form. When the upstream Hippo signal is absent, YAP undergoes dephosphorylation and enters the nucleus. In the nucleus, YAP acts as a transcriptional coactivator to promote cell proliferation. We investigated the role of YAP in AGR2mediated improvement of intestinal mucosal barrier injury by examining the expression and phosphorylation level of YAP protein. The results showed that, in the model of rhTNF- $\alpha$-induced intestinal mucosal barrier injury, the expression of YAP protein was significantly reduced, while the phosphorylation level of YAP was significantly increased. The YAP protein was mainly distributed in the cytoplasm and lacked transcriptional activity. Overexpression of AGR2 upregulated YAP protein expression $(p<0.01)$ and promoted the translocation of YAP into the nucleus (Figures 3(a) and 3(b)). The above experiments demonstrate that the inhibition of the rhTNF- $\alpha$-mediated intestinal mucosal barrier injury by AGR2 was accompanied by the activation of YAP protein.

To further verify the role of YAP in AGR2-mediated promotion of the repair of intestinal mucosal injury, AGR2 and control plasmids were pretransfected into Caco- 2 cells in a Transwell chamber. Twenty-four hours later, the YAPspecific inhibitor verteporfin $(1 \mu \mathrm{g} / \mathrm{ml})$ was added to the cells, and $2 \mathrm{~h}$ later, rhTNF- $\alpha$ was added. After $48 \mathrm{~h}$, the TEER and yellow fluorescence transmittance were measured to determine the changes in cell membrane permeability. Cell proliferation activity was examined by the CCK- 8 method, and barrier healing was examined by the scratch assay. The results showed that the protective effect of AGR2 on the intestinal barrier was significantly weakened after the addition of verteporfin, which manifested as increased cell membrane permeability, reduced cell proliferation, and a significantly decreased mucosal healing ability (Figures 3(c)-3(f)).

\section{Discussion}

The results of this study showed that the expression of AGR2 in intestinal mucosal epithelial cells was significantly higher in UC patients than in the normal control group. Moreover, the difference in AGR2 expression was positively correlated with Ki67 expression (a marker of the activity of intestinal epithelial cell proliferation) and negatively correlated with the degree of endoscopic mucosal damage. These results indicate that AGR2 might have a protective effect on the mucosa by promoting the proliferation of intestinal epithelial cells. In vitro experiments showed that AGR2 promoted the proliferation and migration of intestinal epithelial cells, thereby inhibiting the intestinal mucosal barrier injury mediated by the inflammatory factor TNF- $\alpha$. This process was accompanied by the activation of the transcription factor YAP. Once YAP activity was inhibited, the protective effect of AGR2 resulting from the promotion of mucosal repair was almost fully abolished.

$\mathrm{UC}$ is a chronic, nonspecific inflammatory disease of the intestinal tract that is prone to relapse. The regenerative dysfunction of intestinal epithelial cells is a typical biological feature of UC. Effective repair of the barrier function of the intestinal mucosa is of great significance to the improvement of UC prognosis and the enhancement of survival rate. Rebuilding or repairing the intestinal epithelial barrier requires three things: migration of intestinal epithelial cells to the injured site, proliferation and differentiation of intestinal epithelial cells to generate new intestinal epithelial monolayer cells, and reconstruction of the cell-cell tight junctions and adhesion functions. Mucosal healing occurs at the molecular level. Its regulation and interaction with inflammatory factors, despite widespread attention, have not been well understood. The main unresolved issue is how to allow the damaged mucosa to be completely reconstructed and healed at an early stage, which would prevent chronic wound healing and the later development into intestinal fibrosis.

As a secretory protein, AGR2 is highly expressed in a variety of cell lines in mammals, including Paneth cells, goblet cells, and Msi-1-positive intestinal stem cells. AGR2 is most highly expressed in the ileum and colon [20]. It is involved in various biological processes, including inflammation, proliferation, apoptosis, and migration [21-24]. In goblet cells, AGR2 forms disulfide bonds with the $\mathrm{N}$ - and $\mathrm{C}$ terminal cysteine-rich regions of the mucin MUC2 through its cysteine residues, thereby participating in the production of mucin [25]. MUC2 is an important component of the mucosal layer covering the surface of the gastrointestinal tract epithelial cells. It acts as the first line of defense against symbiotic bacteria and pathogenic bacteria. In an AGR2knockout mouse model, injury of the intestinal tract cannot be effectively repaired. However, the specific molecular mechanism has not been elucidated. The AGR2 mRNA level is significantly increased in the intestinal tissues of UC patients [26].

To clarify the role of AGR2 in mucosal repair in UC, we first conducted immunohistochemical staining to examine the difference in AGR2 protein expression in intestinal tissues between UC patients and the normal control group. 


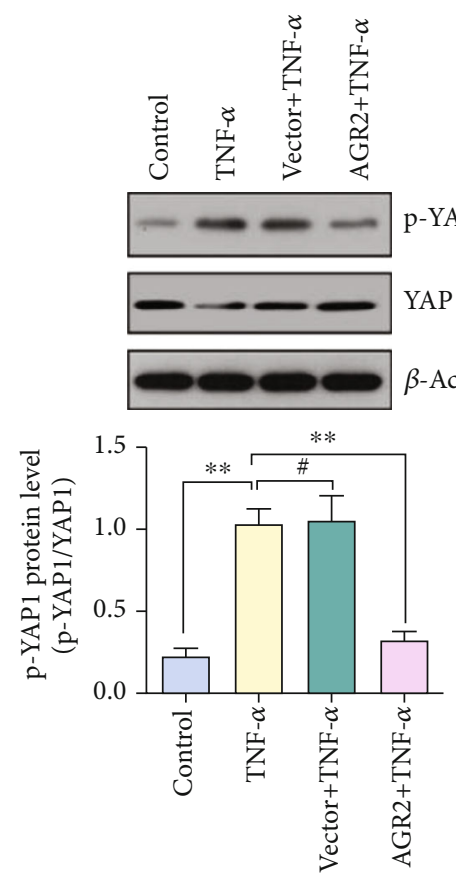

(a)
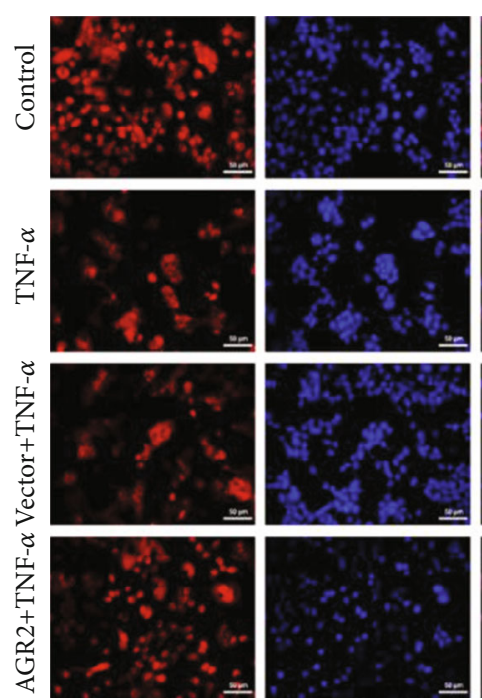

YAP

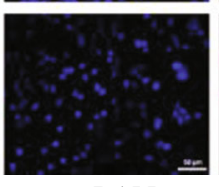

DAPI
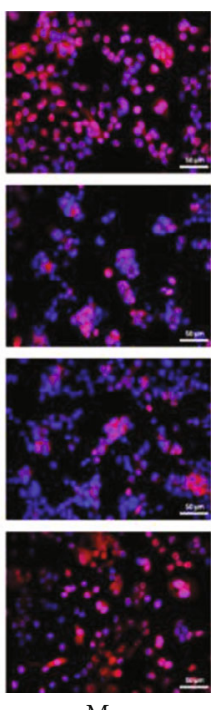

Merge

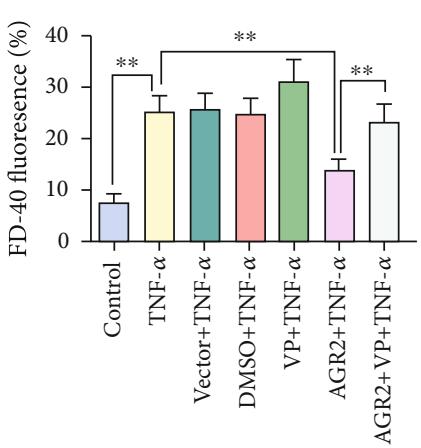

(c)

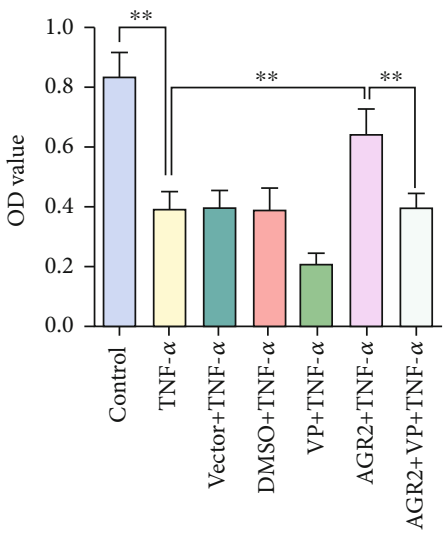

(e)
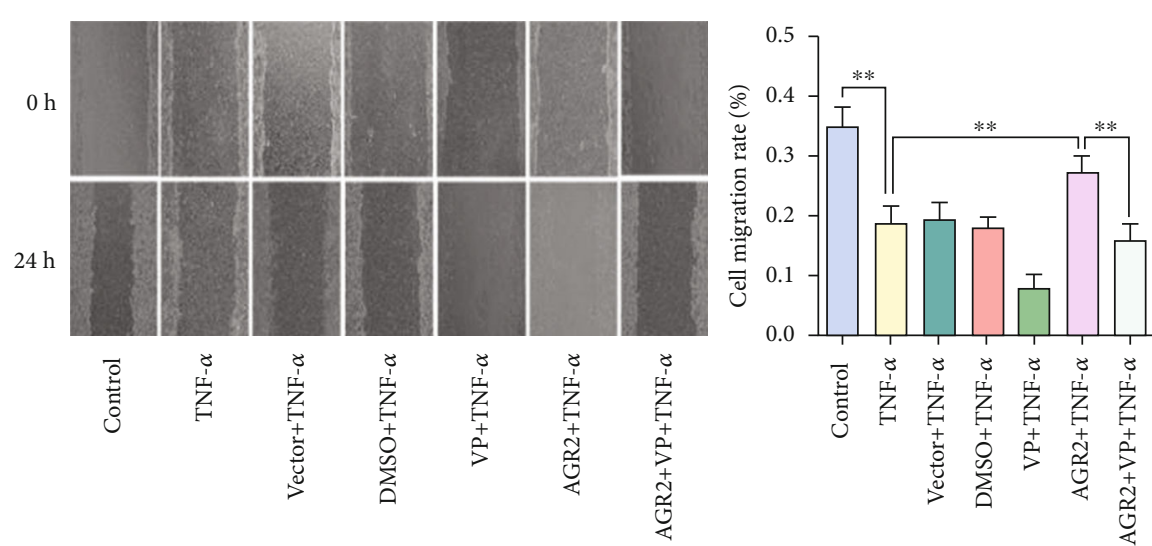

(f)

FIgURE 3: AGR2 promotes the repair of intestinal mucosal barrier injury. (a) The YAP protein expression and phosphorylation levels as determined by Western blot analysis are shown. (b) Distribution of YAP detected by immunofluorescence staining. (c) The epithelial permeability was detected by evaluating TEER. (d) The epithelial permeability was detected by FD-40 flux. (e) Viability of the Caco-2 cells as detected by CCK- 8 assay. (f) Migratory ability of the Caco- 2 cells as evaluated by wound healing assay. The data are presented as the mean \pm SD, ${ }^{*} p>0.05,{ }^{*} p<0.05,{ }^{* *} p<0.01$. 
The results showed that the AGR2-positive staining rate was significantly higher in the intestinal tissues of UC patients than in the normal control group, which is consistent with the results of Maurel et al. [27]. The positive staining was located close to the intestinal stem cells and was distributed in both the cytoplasm and the nucleus of intestinal mucosal epithelial cells. Moreover, the positive staining was positively correlated with the expression of the cell proliferation marker Ki67 and negatively correlated with the Rachmilewitz endoscopic score. These results demonstrate that AGR2 might be important in the proliferation of intestinal mucosal epithelial cells, thereby mediating barrier repair after intestinal mucosal injury. In addition, AGR2 may be used as a new biomarker to monitor endoscopic mucosal conditions in UC patients.

AGR2 plays an important role in the homeostasis, inflammation, and repair of intestinal mucosal epithelial cells. To further understand its specific mode of action, we used in vitro-cultured Caco- 2 cells to simulate the intestinal epithelial barrier and explored the potential molecular mechanism at the cellular level. The results showed that overexpression of AGR2 inhibited the rhTNF- $\alpha$-induced decline in cell proliferation activity. The two main factors leading to cell proliferation disorders are cell cycle arrest and increased apoptosis [28-30]. Uncontrolled cell cycle progression is one of the causes of intestinal epithelial barrier defects $[31,32]$. Among the cell cycle regulation checkpoints, the one between G1 and S phases is the most important. The abnormal expression of the proteins that carry out this checkpoint may lead to a decrease in the proliferation activity of intestinal epithelial cells. In this study, the percentage of cells in the G0/G1 phase was significantly increased after $\operatorname{rhTNF}-\alpha$ stimulation, whereas the percentage of cells in S and G2/M phases was decreased. These findings are consistent with the results of previous studies $[33,34]$. AGR2 inhibited the rhTNF- $\alpha$ induced cell cycle arrest, allowing a smooth transition from the G1 phase to the S phase. To further clarify the molecular mechanism by which AGR2 affects cell cycle, we examined the expression of the key cell cycle protein cyclin D1 by Western blot. The results showed that the expression of cyclin D1 protein was significantly decreased after the addition of rhTNF- $\alpha$. In contrast, overexpression of AGR2 inhibited the TNF- $\alpha$-induced decrease in cyclin D1 protein expression. The above results demonstrate that AGR2 accelerated the transition from $\mathrm{G} 1$ to $\mathrm{S}$ phase by regulating the expression of cyclin D1.

Apoptosis is another major factor leading to disordered repair of intestinal epithelial barrier injury. Excessive apoptosis of intestinal epithelial cells will result in a decrease in the number of cells with tight junctions and an aggravation of intestinal tissue damage [35-37]. We found that apoptosis increased significantly after rhTNF- $\alpha$ stimulation. This finding is consistent with the results of previous studies [38, 39]. AGR2 inhibited the rhTNF- $\alpha$-mediated apoptosis. The above results demonstrate that the high expression of AGR2 in the intestinal mucosa mediates the protective effect against colitis by promoting epithelial cell proliferation and inhibiting apoptosis.

Multiple signaling pathways are involved in the repair of intestinal mucosal barrier injury, such as those of Notch, Hippo/YAP, epidermal growth factor receptor (EGFR), and transforming growth factor beta (TGF- $\beta$ ) [40-45]. YAP, an effector protein of the Hippo pathway, was recently discovered to be important in the maintenance of intestinal barrier function in IBD. YAP is mainly located in the nucleus of Lgr $5^{+}$cells. It is expressed in both the ileum and colon and plays an important role in the maintenance of intestinal stem cell function and in tissue regeneration [19, 46, 47]. In a mouse model of dextran sulfate sodium-induced colitis, knockout of the YAP gene inhibited tissue regeneration, resulting in weight loss and significantly increased mortality rate [48]. Based on the similar biological role of AGR2 and YAP in intestinal epithelial cells, we suspected that there was an internal connection between the two proteins. To clarify the interaction between AGR2 and YAP during the repair of mucosal damage, we constructed a model of intestinal mucosal barrier injury in vitro. AGR2 improved the $\operatorname{rhTNF}-\alpha$-mediated intestinal mucosal barrier injury, and YAP was activated in this process. The protective effect of AGR2, which was resulted from the promotion of mucosal repair, was significantly attenuated after adding the specific inhibitor verteporfin, which was manifested as a decline in cell proliferative ability. Another important step in the repair of intestinal mucosal barrier injury is epithelial reconstruction, namely, the migratory ability of epithelial cells. Cell migration is one of the basic characteristics of many cells. Cell migration plays an important role in various physiological and pathological conditions, such as injury repair and immune response $[49,50]$. We performed the scratch-wound healing experiment to examine the effect of AGR2 on cell migratory ability in rhTNF$\alpha$-induced intestinal epithelial barrier injury. The results showed that the migratory ability of the cells was significantly reduced after rhTNF- $\alpha$ stimulation. In contrast, pretransfection with AGR2 plasmid significantly enhanced cell migratory ability. After adding verteporfin, the ability of AGR2 to promote cell migration was significantly suppressed.

In summary, the high expression of AGR2 in the intestinal mucosa protects against colon inflammation by promoting epithelial cell proliferation and migration. The highly expressed AGR2 activates YAP, a downstream effector molecule of the Hippo pathway, thereby promoting the recovery of intestinal mucosal barrier function.

\section{Abbreviations}

AGR2: Anterior gradient protein 2

IBD: Inflammatory bowel disease

TNF- $\alpha$ : Tumor necrosis factor-alpha

YAP: Yes-associated protein

CD: Crohn's disease

PDI: Protein disulfide isomerase

TEER: Transepithelial electrical resistance

WBC: White blood cell

Hb: Hemoglobin

PLT: Platelet

Alb: Albumin

CRP: C-reactive protein

ESR: Erythrocyte sedimentation rate 
UC: $\quad$ Ulcerative colitis.

\section{Data Availability}

The data used to support the findings of this study are available from the corresponding author upon request.

\section{Ethical Approval}

This study conformed with the Declaration of Helsinki and was implemented strictly following a protocol approved by the Medical Ethics Committee of Shengjing Hospital, China Medical University.

\section{Consent}

All patients and participants were informed of the study and voluntarily signed informed consent.

\section{Conflicts of Interest}

The authors declare that there are no conflicts of interest regarding the publication of this paper.

\section{Acknowledgments}

This work was supported by a grant from the Doctoral Scientific Research Foundation of Liaoning Province of China (No. 2020-BS-092).

\section{References}

[1] L. W. Peterson and D. Artis, "Intestinal epithelial cells: regulators of barrier function and immune homeostasis," Nature Reviews. Immunology, vol. 14, no. 3, pp. 141-153, 2014.

[2] R. Garcia-Carbonell, S. J. Yao, S. Das, and M. Guma, "Dysregulation of intestinal epithelial cell RIPK pathways promotes chronic inflammation in the IBD gut," Frontiers in Immunology, vol. 10, p. 1094, 2019.

[3] C. H. F. Hansen, C. S. Larsen, H. O. Petersson et al., "Targeting gut microbiota and barrier function with prebiotics to alleviate autoimmune manifestations in NOD mice," Diabetologia, vol. 62, no. 9, pp. 1689-1700, 2019.

[4] G. T. Ho, J. A. Cartwright, E. J. Thompson, C. C. Bain, and A. G. Rossi, "Resolution of inflammation and gut repair in IBD: translational steps towards complete mucosal healing," Inflammatory Bowel Diseases, vol. 26, no. 8, pp. 1131-1143, 2020.

[5] A. T. Blikslager, A. J. Moeser, J. L. Gookin, S. L. Jones, and J. Odle, "Restoration of barrier function in injured intestinal mucosa," Physiological Reviews, vol. 87, no. 2, pp. 545-564, 2007.

[6] H. Seno, H. Miyoshi, S. L. Brown, M. J. Geske, M. Colonna, and T. S. Stappenbeck, "Efficient colonic mucosal wound repair requires Trem 2 signaling," Proceedings of the National Academy of Sciences of the United States of America, vol. 106, no. 1, pp. 256-261, 2009.

[7] F. Zhao, R. Edwards, D. Dizon et al., "Disruption of Paneth and goblet cell homeostasis and increased endoplasmic reticulum stress in Agr 2-/- mice," Developmental Biology, vol. 338, no. 2, pp. 270-279, 2010.
[8] X. Ye and M. Sun, "AGR2 ameliorates tumor necrosis factor$\alpha$-induced epithelial barrier dysfunction via suppression of NF- $\kappa$ B p 65 -mediated MLCK/p-MLC pathway activation," International Journal of Molecular Medicine, vol. 39, no. 5, pp. 1206-1214, 2017.

[9] Subspecialty Group of Gastroenterology tSoPCMA and Subspecialty Group of Clinical Nutrition tSoPCMA, "Expert consensus on the diagnosis and management of pediatric inflammatory bowel disease," Zhonghua $\mathrm{Er} \mathrm{Ke} \mathrm{Za} \mathrm{Zhi,}$ vol. 57, pp. 501-507, 2019.

[10] A. Levine, A. Griffiths, J. Markowitz et al., "Pediatric modification of the Montreal classification for inflammatory bowel disease: the Paris classification," Inflammatory Bowel Diseases, vol. 17, no. 6, pp. 1314-1321, 2011.

[11] C. Ha and A. Kornbluth, "Mucosal healing in inflammatory bowel disease: where do we stand?," Current Gastroenterology Reports, vol. 12, no. 6, pp. 471-478, 2010.

[12] K. Kamaradova, H. Vosmikova, K. Rozkosova, A. Ryska, I. Tacheci, and J. Laco, "Morphological, immunohistochemical and molecular features of inflammatory bowel disease associated colorectal carcinoma and associated mucosal lesions - single institution experience," Pathology, Research and Practice, vol. 215 , no. 4 , pp. 730-737, 2019.

[13] L. Dumartin, H. J. Whiteman, M. E. Weeks et al., "AGR2 is a novel surface antigen that promotes the dissemination of pancreatic cancer cells through regulation of cathepsins B and D," Cancer Research, vol. 71, no. 22, pp. 7091-7102, 2011.

[14] M. Nighot, R. Al-Sadi, S. Guo et al., "Lipopolysaccharideinduced increase in intestinal epithelial tight permeability is mediated by toll-like receptor 4 /myeloid differentiation primary response 88 (MyD88) activation of myosin light chain kinase expression," The American Journal of Pathology, vol. 187, no. 12, pp. 2698-2710, 2017.

[15] R. Yang, T. Harada, J. Li et al., "Bile modulates intestinal epithelial barrier function via an extracellular signal related kinase 1/2 dependent mechanism," Intensive Care Medicine, vol. 31, no. 5, pp. 709-717, 2005.

[16] K. Brandl, S. Rutschmann, X. Li et al., "Enhanced sensitivity to DSS colitis caused by a hypomorphic Mbtps 1 mutation disrupting the ATF6-driven unfolded protein response," Proceedings of the National Academy of Sciences of the United States of America, vol. 106, no. 9, pp. 3300-3305, 2009.

[17] S. Sabui, J. A. Bohl, R. Kapadia et al., "Role of the sodiumdependent multivitamin transporter (SMVT) in the maintenance of intestinal mucosal integrity," American Journal of Physiology. Gastrointestinal and Liver Physiology, vol. 311, no. 3, pp. G561-G570, 2016.

[18] J. Li, W. Hu, and Q. Lan, "The apoptosis-resistance in t-AUCBtreated glioblastoma cells depends on activation of Hsp 27," Journal of Neuro-Oncology, vol. 110, no. 2, pp. 187-194, 2012.

[19] F. Deng, L. Peng, Z. Li et al., "YAP triggers the Wnt/ $\beta$-catenin signalling pathway and promotes enterocyte self-renewal, regeneration and tumorigenesis after DSS-induced injury," Cell Death \& Disease, vol. 9, no. 2, p. 153, 2018.

[20] J. Chang, M. R. Chance, C. Nicholas et al., "Proteomic changes during intestinal cell maturation in vivo," Journal of Proteomics, vol. 71, no. 5, pp. 530-546, 2008.

[21] S. Tian, J. Hu, K. Tao et al., "Secreted AGR2 promotes invasion of colorectal cancer cells via Wnt11-mediated non-canonical Wnt signaling," Experimental Cell Research, vol. 364, no. 2, pp. 198-207, 2018. 
[22] L. Sommerova, E. Ondrouskova, B. Vojtesek, and R. Hrstka, "Suppression of AGR2 in a TGF- $\beta$-induced Smad regulatory pathway mediates epithelial-mesenchymal transition," $B M C$ Cancer, vol. 17, no. 1, p. 546, 2017.

[23] D. Wodziak, A. Dong, M. F. Basin, and A. W. Lowe, "Anterior gradient 2 (AGR2) induced epidermal growth factor receptor (EGFR) signaling is essential for murine pancreatitisassociated tissue regeneration," PLoS One, vol. 11, no. 10, article e0164968, 2016.

[24] Q. Zhu, H. B. Mangukiya, D. S. Mashausi et al., "Anterior gradient 2 is induced in cutaneous wound and promotes wound healing through its adhesion domain," The FEBS Journal, vol. 284, no. 17, pp. 2856-2869, 2017.

[25] S. W. Park, G. Zhen, C. Verhaeghe et al., "The protein disulfide isomerase AGR2 is essential for production of intestinal mucus," Proceedings of the National Academy of Sciences of the United States of America, vol. 106, no. 17, pp. 6950-6955, 2009.

[26] G. F. Camarillo, E. I. Goyon, R. B. Zuniga, L. A. S. Salas, A. E. P. Escarcega, and J. K. Yamamoto-Furusho, "Gene expression profiling of mediators associated with the inflammatory pathways in the intestinal tissue from patients with ulcerative colitis," Mediators of Inflammation, vol. 2020, Article ID 9238970 , 11 pages, 2020.

[27] M. Maurel, J. Obacz, T. Avril et al., "Control of anterior GRadient 2 (AGR2) dimerization links endoplasmic reticulum proteostasis to inflammation," EMBO Molecular Medicine, vol. 11, no. 6, 2019.

[28] S. Dalton, "Linking the cell cycle to cell fate decisions," Trends in Cell Biology, vol. 25, no. 10, pp. 592-600, 2015.

[29] L. R. Pack, L. H. Daigh, and T. Meyer, "Putting the brakes on the cell cycle: mechanisms of cellular growth arrest," Current Opinion in Cell Biology, vol. 60, pp. 106-113, 2019.

[30] K. S. Twayana and P. Ravanan, "Eukaryotic cell survival mechanisms: disease relevance and therapeutic intervention," Life Sciences, vol. 205, pp. 73-90, 2018.

[31] K. Gronke, P. P. Hernández, J. Zimmermann et al., "Interleukin-22 protects intestinal stem cells against genotoxic stress," Nature, vol. 566, no. 7743, pp. 249-253, 2019.

[32] K. E. Cunningham, E. A. Novak, G. Vincent et al., "Calcium/calmodulin-dependent protein kinase IV (CaMKIV) activation contributes to the pathogenesis of experimental colitis via inhibition of intestinal epithelial cell proliferation," The FASEB Journal, vol. 33, no. 1, pp. 1330-1346, 2019.

[33] Q. Yan, X. Chen, H. Gong et al., "Delivery of a TNF- $\alpha$-derived peptide by nanoparticles enhances its antitumor activity by inducing cell-cycle arrest and caspase-dependent apoptosis," The FASEB Journal, vol. 32, no. 12, pp. 6948-6964, 2018.

[34] W. Chen, W. Wang, L. Chen et al., "Long-term G1 cell cycle arrest in cervical cancer cells induced by co-immobilized TNF- $\alpha$ plus IFN- $\gamma$ polymeric drugs," Journal of Materials Chemistry B, vol. 6, no. 2, pp. 327-336, 2018.

[35] C. Zhang, W. Shu, G. Zhou et al., “Anti-TNF- $\alpha$ Therapy Suppresses Proinflammatory Activities of Mucosal Neutrophils in Inflammatory Bowel Disease," Mediators of Inflammation, vol. 2018, Article ID 3021863, 12 pages, 2018.

[36] R. Garcia-Carbonell, J. Wong, J. Y. Kim et al., "Elevated A20 promotes TNF-induced and RIPK1-dependent intestinal epithelial cell death," Proceedings of the National Academy of Sciences, vol. 115, no. 39, pp. E9192-E9200, 2018.
[37] U. Billmeier, W. Dieterich, M. F. Neurath, and R. Atreya, "Molecular mechanism of action of anti-tumor necrosis factor antibodies in inflammatory bowel diseases," World Journal of Gastroenterology, vol. 22, no. 42, pp. 9300-9313, 2016.

[38] J. Pott, A. M. Kabat, and K. J. Maloy, "Intestinal Epithelial Cell Autophagy Is Required to Protect against TNF- Induced Apoptosis during Chronic Colitis in Mice," Cell Host Microbe, vol. 23, no. 2, pp. 191-202.e4, 2018.

[39] Y. Yuan, D. Ding, N. Zhang et al., “TNF- $\alpha$ induces autophagy through ERK1/2 pathway to regulate apoptosis in neonatal necrotizing enterocolitis model cells IEC-6," Cell Cycle, vol. 17, no. 11, pp. 1390-1402, 2018.

[40] C. A. Lindemans, M. Calafiore, A. M. Mertelsmann et al., "Interleukin-22 promotes intestinal-stem-cell-mediated epithelial regeneration," Nature, vol. 528, no. 7583, pp. 560-564, 2015.

[41] J.-. C. Lin, J.-. Q. Wu, F. Wang et al., "QingBai decoction regulates intestinal permeability of dextran sulphate sodiuminduced colitis through the modulation of notch and NF- $\kappa \mathrm{B}$ signalling," Cell Proliferation, vol. 52, no. 2, article e12547, 2019.

[42] X. Zhou, W. Li, S. Wang et al., "YAP aggravates inflammatory bowel disease by regulating M1/M2 macrophage polarization and gut microbial homeostasis," Cell Reports, vol. 27, no. 4, pp. 1176-1189.e5, 2019.

[43] T. E. Riehl, D. Alvarado, X. Ee, M. A. Ciorba, and W. F. Stenson, "Hyaluronic acid promotes Lgr 5(+) stem cell proliferation and crypt fission through TLR4 and PGE2 transactivation of EGFR," American Journal of Physiology. Gastrointestinal and Liver Physiology, vol. 319, no. 1, pp. G63-G73, 2020.

[44] S. Ihara, Y. Hirata, and K. Koike, "TGF- $\beta$ in inflammatory bowel disease: a key regulator of immune cells, epithelium, and the intestinal microbiota," Journal of Gastroenterology, vol. 52, no. 7, pp. 777-787, 2017.

[45] S. Sedda, I. Marafini, V. Dinallo, D. Di Fusco, and G. Monteleone, "The TGF- $\beta /$ Smad system in IBD pathogenesis," Inflammatory Bowel Diseases, vol. 21, no. 12, pp. 29212925, 2015.

[46] S. Yui, L. Azzolin, M. Maimets et al., "YAP/TAZ-dependent reprogramming of colonic epithelium links ECM remodeling to tissue regeneration," Cell Stem Cell, vol. 22, no. 1, pp. 3549.e7, 2018.

[47] K. Taniguchi, L. W. Wu, S. I. Grivennikov et al., "A gp130-SrcYAP module links inflammation to epithelial regeneration," Nature, vol. 519, no. 7541, pp. 57-62, 2015.

[48] H. B. Kim, M. Kim, Y. S. Park et al., "Prostaglandin E2 activates YAP and a positive-signaling loop to promote colon regeneration after colitis but also carcinogenesis in mice," Gastroenterology, vol. 152, no. 3, pp. 616-630, 2017.

[49] J. M. Golden, O. H. Escobar, M. V. L. Nguyen et al., "Ursodeoxycholic acid protects against intestinal barrier breakdown by promoting enterocyte migration via EGFR- and COX-2dependent mechanisms," American Journal of Physiology. Gastrointestinal and Liver Physiology, vol. 315, no. 2, pp. G259G271, 2018.

[50] X. Sun, Q. Yang, C. J. Rogers, M. Du, and M. J. Zhu, “AMPK improves gut epithelial differentiation and barrier function via regulating Cdx2 expression," Cell Death and Differentiation, vol. 24, no. 5, pp. 819-831, 2017. 